\title{
Cysteine Linkages Accelerate Electron Flow through Tetra-Heme Protein STC
}

\author{
Xiuyun Jiang, ${ }^{\dagger}$ Zdenek Futera, ${ }^{\dagger}$ Md. Ehesan Ali $,{ }^{\dagger},{ }^{\dagger}$ Fruzsina Gajdos, ${ }^{\dagger}$ Guido F. von Rudorff, ${ }^{\dagger}$ Antoine \\ Carof, ${ }^{\dagger}$ Marian Breuer, ${ }^{\dagger, \S}$ Jochen Blumberger, ${ }^{\dagger}, \|$ ** \\ ${ }^{\dagger}$ Department of Physics and Astronomy, University College London, London WC1E 6BT, U.K. \\ "I Institute for Advanced Study, Technische Universität München, Lichtenbergstrasse 2a, D-85748 Garching, Germany
}

Supporting Information Placeholder

\begin{abstract}
Multi-heme proteins have attracted much attention recently due to their prominent role in mediating extracellular electron transport (ET), but one of their key fundamental properties, the rate constants for ET between the constituent heme groups, have so far evaded experimental determination. Here we report the set of heme-heme theoretical ET rate constants that define electron flow in the tetra-heme protein STC by combining a novel projectoroperator diabatization approach for electronic coupling calculation with molecular dynamics simulation of ET free energies. On the basis of our calculations, we find that the protein limited electron flux through STC in the thermodynamic downhill direction (heme $1 \rightarrow 4$ ) is $\approx 3.10^{6} \mathrm{~s}^{-1}$. We find that cysteine linkages inserting in the space between the two terminal heme pairs 1-2 and 3-4 significantly enhance the overall electron flow, by a factor of about 37 , due to weak mixing of the sulfur $3 p$ orbital with the Fe-heme $d_{\pi}$ orbitals. While the packing density model, and to a higher degree, the pathway model of biological ET partly capture the predicted rate enhancements, our study highlights the importance of the atomistic and chemical nature of the tunneling medium at short biological tunneling distances. Cysteine linkages are likely to enhance electron flow also in the larger deca-heme proteins MtrC and MtrF, where hemeheme motifs with sub-optimal edge-to-edge distances are used to shuttle electrons in multiple directions.
\end{abstract}

Certain microbes have developed an extraordinary type of respiration in response to conditions of low oxygen concentration. In a process known as extracellular respiration they metabolize (oxidize) organic matter inside the cell and transport the electrons generated across the cell envelope to the cell's exterior for reduction of insoluble transitionmetal oxides ${ }^{1}$ or interspecies electron transfer. ${ }^{2}$ Key to extracellular respiration are conducting multi-heme cytochromes which are arranged into molecular "nanowires" capable of transporting electrons over micrometer distances. ${ }^{3-6}$ From a technological point of view, these enzymes may represent a new category of bioorganic conductive materials for novel bionanotechnological applications ${ }^{7,8}$ such as electronic communication, signaling and sensing with bacterial cells, non-toxic implantable bioelectronics devices or even artificial skin.

In order to fully explore the potential and help design multi-heme proteins for future bioelectronic applications, it is vital to characterize and understand the electron transport properties of these fascinating proteins on a molecular level of detail. In previous theoretical work our group has investigated the thermodynamics, ${ }^{9}$ kinetics ${ }^{10}$ and mechanism ${ }^{11}$ for electron transport in the deca-heme protein MtrF confirming earlier suggestions ${ }^{12-14}$ that ET in this structure occurs via step-wise hopping between neighboring $\mathrm{Fe}^{2+} /$ $\mathrm{Fe}^{3+}$ heme pairs. Other mechanisms such as superexchange and flickering resonance ${ }^{15}$ were deemed uncompetitive. However, the computed protein-limited electron flux through $\mathrm{MtrF}^{10}$ underestimated the experimentally determined currents ${ }^{16,17}$ by about two orders of magnitude. This suggests that the rate-determining ET step(s) in this protein might be higher than previously predicted.

Here we investigate ET in the smaller tetra-heme cytochrome $\operatorname{STC}^{18-20}$ (see Figure 1), one of the smallest representatives of the multi-heme protein family with an experimentally resolved structure. Although this protein is thought to function as a soluble electron carrier rather than a tetra-heme wire, ${ }^{20}$ it features very similar heme-heme motifs as the deca-heme proteins $\mathrm{MtrF}^{3}$ and MtrC. ${ }^{5} \mathrm{We}$ find that cysteine linkages significantly accelerate ET between the two T-shaped heme pairs at the electron input and output sites of STC that limit the electron flow through the protein (heme pairs 1-2 and 3-4 in Figure 1). This effect is traced back to weak mixing of the sulfur $3 p$ orbital with the redox active Fe-heme frontier orbitals, see inset in Figure 1 , which leads to a marked increase in heme-heme electronic couplings. This mechanism may permit the formation of heme-heme motifs with relatively large heme edge-to-edge separation distances without sacrificing too much ET speed. Preliminary analysis of the cysteine linkages in $\mathrm{MtrF}$ and $\mathrm{MtrC}$ indicate that a similar rateenhancing effect, not included in the previous calculations, ${ }^{10}$ may be present in these proteins as well. In the following we detail calculations of the two key parameters determining the heme-heme ET rates, electronic coupling 


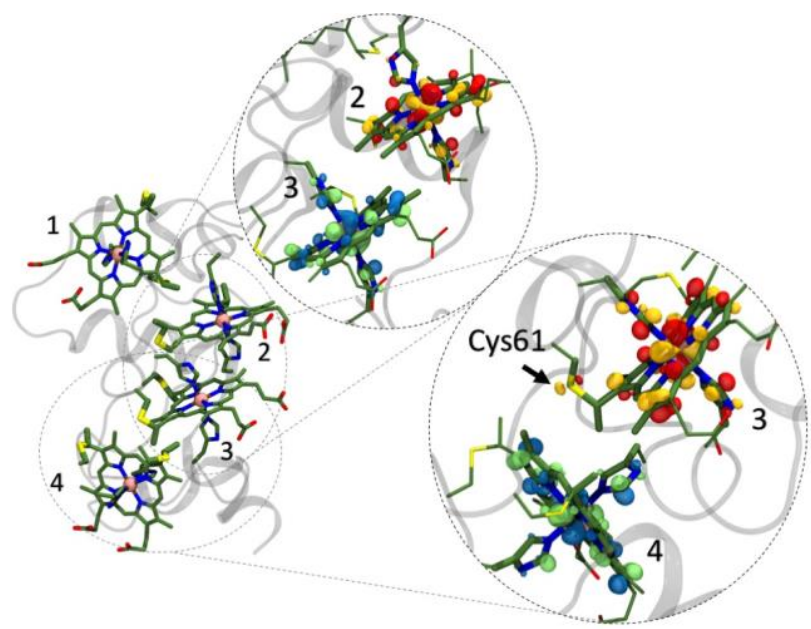

Figure 1: Crystal structure of the STC protein from S. onei-

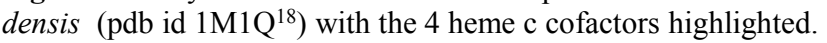
The T-shaped heme pair 3-4 with the cysteine 61 linkage (marked by arrow), and the stacked heme pair 2-3 are shown enlarged. Isosurfaces of the Fe-heme frontier orbital pairs mediating the electron transfer are superimposed. Colour code of atoms: Fe: pink, S: yellow, O; red, N: blue, C: green.

and reorganization free energy, before discussing the predicted electron flux in STC.

ET in multi-heme proteins occurs via sequential electron hopping between neighboring, low-spin $\mathrm{Fe}^{2+}$ and $\mathrm{Fe}^{3+}$-heme cofactors. ${ }^{11}$ The effective electronic coupling matrix element $\left|H_{\mathrm{ab}}\right|$ between the redox active frontier molecular orbitals of the heme groups (composed of Fe-3d and heme ring orbitals) are calculated using the projector-operator diabatization (POD) approach, ${ }^{21}$ see supporting information (SI) for details. Here we investigate the importance of the heme side chains on electronic coupling by successively increasing the size of the quantum-mechanical (QM) heme model (see Figures S1-S2 and Table S1 in the SI for a description of all models investigated). We first present our findings for ET between heme pair 3-4, which forms a Tshaped motif as shown in Figure 1.

The simplest heme model 1, Fe-porphin axially ligated by two methyl-imidazoles and all heme side chains replaced with hydrogen atoms, gives rather small couplings of less than $1 \mathrm{meV}$, see Figure 2A (blue solid line). Importantly, upon inclusion of the side chains inserting in the space between the two heme groups (model 2), we obtain a significant increase in electronic coupling by a factor of 3.2. Further additions of side chains pointing away from the electron transfer partner (models 3 and $\mathbf{4}$ ), have only a minor effect. The coupling for the largest model investigated (model 5), comprised of all side chains including the propionates, differs by less than $2 \%$ compared to model 2 indicating that coupling is well converged with respect to system size for model 2. A similar trend is observed for heme pair 1-2 which forms a T-shaped motif at the opposite terminus of the protein (Figure S3).

We find that the strong increase in electronic coupling between heme pair 3-4 (1-2) is due to Cys 61 (18). In Figure 1 we show one of the frontier orbital pair combinations that contribute to electronic coupling in pair 3-4. One can clearly see that the sulfur $3 p$ orbital of Cys 61 mixes with
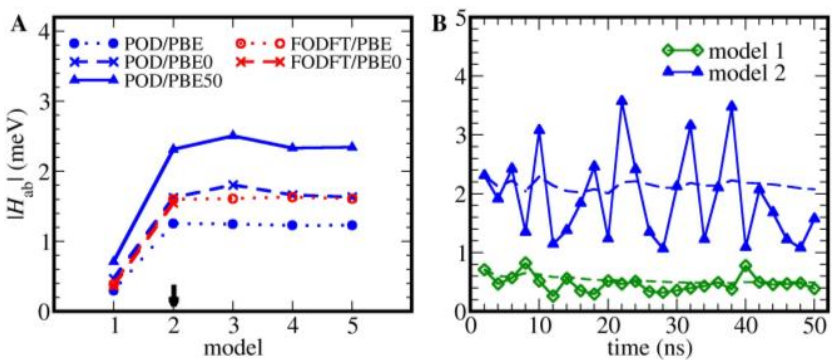

Figure 2: $\left|H_{\mathrm{ab}}\right|$ values for heme pair 3-4. (A) Convergence with respect to the model size, as obtained with POD and FODFT methods for different density functional theory (DFT) functionals. PBE50 means $50 \%$ of $\mathrm{PBE}^{22}$ exchange is replaced by the exact Hartree-Fock exchange. The sharp increase in $\left|H_{\mathrm{ab}}\right|$ from $\mathbf{1}$ to $\mathbf{2}$ is due to inclusion of the cysteine linkages in model 2 . The bold arrow indicates the final model chosen for presented rate calculations. (B) Thermal fluctuations of $\left|H_{\mathrm{ab}}\right|$ at POD/PBE50 level along a MD trajectory using model 1 (green) and model 2 (blue). Dashed lines indicate the accumulated average $\left\langle\left|H_{\mathrm{ab}}\right|^{2}>^{1 / 2}\right.$.

the Fe-heme frontier orbitals of heme 3, though relatively weakly as the density of states of sulfur peaks more than 1 $\mathrm{eV}$ below the Fe-3d states. Nonetheless, the mixing is sufficiently strong to modify the tail of the Fe-heme frontier orbitals leading to an increase in overlap with the Fe-heme frontier orbitals of heme 4 and consequently to an increase in electronic coupling. By contrast, no cysteine-mediated increase in coupling is observed for heme pair 2-3 in the middle of the protein. The two heme rings form a stacked motif and approach one another at van der Waals distance with the cysteine linkages oriented nearly perpendicular to the heme planes (see Figure 1).

Our observation that the cysteine linkages increase electronic coupling in heme pairs 3-4 and 1-2 is robust with respect to (i) the fraction of Hartree-Fock exchange (HFX) used in the exchange-correlation functional (ii) the method used for electronic coupling calculation (iii) thermal protein fluctuations. While absolute couplings are sensitive to the fraction of HFX used, a strong increase upon inclusion of cysteine linkages in the model is observed in all calculations (see Figure 2A, blue lines). An alternative method for the electronic coupling calculation based on DFT fragment orbitals (FODFT) ${ }^{23-25}$ gives results very similar to POD (see Figure 2A, red lines and Table S2). To take into account thermal fluctuations we have sampled $H_{\boldsymbol{a} \boldsymbol{b}}$ along classical molecular dynamics (MD) trajectories of the protein at $300 \mathrm{~K}$ (see SI for details). We find that the average, $\left\langle\left|H_{a b}\right|^{2}\right\rangle^{\mathbf{1} / \mathbf{2}}$, for heme pair 3-4 is a factor of 4.2 larger when the cysteine linkages are included (see Figure 2B), in line with the result obtained above for a single configuration. The corresponding increase in coupling for the other $\mathrm{T}$ shaped heme pair 1-2, a factor of 7.3, is even more pronounced than for heme pair 3-4. The stronger effect is consistent with the average heme edge-to-edge separation being larger and the average sulfur-to-heme edge distance being smaller in heme pair 1-2 than in 3-4 (see Table S3).

For calculation of heme-heme ET rate constants, reorganization free energy $\lambda$ and free energy difference (or driving force) $\Delta G$ are needed in addition to electronic couplings. Here, the reorganization free energies for each ET step are obtained from the shift of the mean vertical energy 
gap in initial and final ET state, using classical MD for thermal sampling and electronically polarizable force field for calculation of the gap energy, ${ }^{11}$ see SI for details. The resultant reorganization free energies $\lambda^{\text {st }}$ ("st" for Stokes shift) fall in the range $0.76-1.08 \mathrm{eV}$ in accord with expectations for proteins with partly solvent-exposed cofactors. ${ }^{11}$ An alternative estimate based on the variance of the energy gap $\left(\lambda^{\text {var }}\right)$ gives very similar values deviating from $\lambda^{\text {st }}$ by no more than $10 \%$. Hence, no evidence is found for application of Matyushov's hypothesis, according to which certain redox proteins reduce ET activation barrier through partial freezing of slow modes (resulting in $\lambda^{\text {st }}<<\lambda^{\text {var }}$ ). ${ }^{26,27}$ For calculation of the ET driving force we used the experimental reduction potentials of the fully reduced protein suitably modified by the experimental heme-heme interactions to take into account the effect of heme occupation on redox potential. ${ }^{20}$ The heme occupations were obtained from electron flux simulation at steady state (see below, SI and Table $\mathrm{S} 4$ for a detailed explanation).

The heme-heme ET rate constants excluding and including the effect of the cysteine linkages on electronic coupling are depicted for the forward $(1 \rightarrow 4$, Figure $3 \mathrm{~A})$ and reverse direction $(4 \rightarrow 1$, Figure $3 \mathrm{~B})$. The two terminal ET steps between hemes 1-2 and 3-4 are clearly rate limiting in the computational model that lacks the cysteine linkages. Their inclusion leads to an increase in the ET rate constants by a factor of 39 and 15 to $3 \times 10^{6} \mathrm{~s}^{-1}$ and $5 \times 10^{6} \mathrm{~s}^{-1}$ for $k_{21}$ and $k_{43}$, respectively, significantly reducing the gap to the fastest ET rate between hemes 2-3, $k_{32}=6 \times 10^{8} \mathrm{~s}^{-1}\left(k_{j i}\right.$ denoting ET rate constant from heme $i$ to heme $j$ ). As the couplings are direction independent, both forward/reverse ET rate exhibit the same enhancement.

The rate constants above are for direct heme-to-heme electron tunneling. We also investigated the possibility of
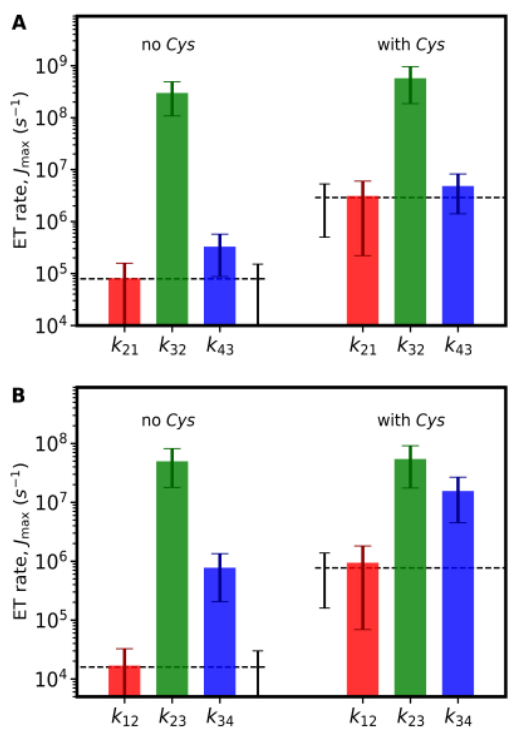

Figure 3: Heme-heme ET rate constants, $k_{j i}$, and the protein limited electron flux, $J_{\max }$ (dashed lines), for the steady-state electron flow in the forward direction from heme 1 to 4 (A) and in the reverse direction (B). The first set of bars in each panel was obtained for model 1, i.e. without cysteine linkages, the second sets included them (model 2,3 for heme pairs 3-4, 1-2). MD-averaged coupling values at POD/PBE50 level of theory were used for calculation of the ET rates. Data taken from column '(SC,p)' in Table S4. heme-heme ET via formation of a positively charged Cyslinkage intermediate, but concluded that this alternative reaction channel is not competitive with direct heme-heme tunneling, see SI for further discussion.

In the following we wish to analyze whether two well established empirical models for biological electron tunneling capture the proposed rate enhancement due to cysteine linkages. We find that the packing density model of Moser and Dutton ${ }^{28}$ predicts similar rate enhancements as our present DFT computations, a factor of 26 and 22 for $k_{21}$ and $k_{43}$, respectively (see SI for details). However, it falls short of predicting a significantly larger increase for $k_{21}$ than for $k_{43}$ because the detailed atomistic geometry of the sulfur linkage leading to a smaller sulfur-to-heme edge distance in pair 2-1 than in 4-3 is not explicitly represented in the packing density approach. The pathway model of Beratan and Onuchic ${ }^{29}$ improves on this issue, giving rate enhancement factors of 102 and 42 for $k_{21}$ and $k_{43}$, respectively (see SI for details). This implies that the ET may be understood in terms of simple through-bond through-space mediation. A similar pathway-specific mediation effect has been found for heme-to-heme ET in cytochrome c oxidase with a methyl group implicated as an essential tunneling mediator. ${ }^{30}$ In this respect it is interesting to note that if we replace the sulfur atom by a $\mathrm{CH}_{2}$ group in our system, the rate enhancement almost disappears in DFT calculations (factor 2), while in the pathway model it remains nearly unchanged (factor 123). Hence, our analysis highlights the importance of not only the atomistic but also the chemical details of the tunneling medium. The latter may be captured by a refined pathway model with chemical specificity.

Finally, we determined the maximum, protein-limited electron flux, $J_{\max }$. To this end, we derived an analytic solution to the master equation for electron flux from heme $i$ to heme $j, J_{j i}=k_{j i} P_{i}\left(1-P_{j}\right)-k_{i j} P_{j}\left(1-P_{i}\right)$, under conditions of steady state flux, $J_{j i}=$ const for all $i, j\left(P_{i}\right.$ is the electron occupation of heme $i$ ). We assumed unlimited electron supply and fast electron injection in one terminal heme and fast ejection from the other, in which case $J_{j i}$ becomes the protein limited flux $J_{\max }$ (see SI for further details). $J_{\max }$, indicated in Figure 3 (dashed lines), is only slightly smaller than the slowest ET rate for a given flow direction. We obtain values of $3 \times 10^{6} \mathrm{~s}^{-1}$ for the forward direction (heme 1 electron input site, heme 4 output site) and $1 \times 10^{6} \mathrm{~s}^{-1}$ for the reverse direction, corresponding to a flux enhancement of a factor of 37 and 48, respectively, due to the presence of the cysteine linkages. Interestingly, the tetra-heme wire conducts almost equally well in both directions as the thermodynamic bias under steady-state conditions occurs for ET between hemes 2 and 3, which is not limiting $J_{\max }$.

To summarize, we have found that the cysteine linkages inserting into the space between heme groups enhance the electronic coupling. In this way, heme pairs forming motifs with larger heme-to-heme edge distances (e.g. co-planar or T-shaped) can exhibit similar ET rates as heme pairs with very short edge-to-edge distance (e.g. stacked orientations). A similar rate-enhancing effect is likely to be present in the decaheme proteins $\mathrm{MtrF}^{3}$ and MtrC. ${ }^{5}$ Structural analysis of $\mathrm{MtrF}$ shows that cysteine linkages bridge the space between heme pairs that were previously identified as rate- 
limiting. ${ }^{10}$ However, these calculations did not take into account the cysteines, which calls for a re-evaluation of the rate-constants in these proteins. Returning to STC, experimental pump-probe measurements on $\mathrm{Ru}$-labeled variants of this proteins are currently ongoing, which we hope will verify the computed heme-heme ET rates reported herein.

\section{ASSOCIATED CONTENT}

The Supporting Information is available free of charge on the ACS Publications website. MD simulation protocols, computational QM models for coupling calculations, calculation of reorganization free energies and electronic couplings, alternative ET channel, packing density and pathway models, derivation of analytic solution to Master equation, explanation of incorporation of heme-heme interactions and protonation of ionizable site in flux calculations.

\section{AUTHOR INFORMATION}

\section{Corresponding Author}

*Email: j.blumberger@ucl.ac.uk

\section{Present Addresses}

* Institute of Nanoscience and Technology, Sector - 64, Phase X, Mohali, India

$\S$ University of Illinois at Urbana-Champaign, Center for the Physics of Living Cells, USA

\section{ACKNOWLEDGMENTS}

We thank Julea Butt, Christopher Hall and Jessica Van Wonderen for illuminating discussions on STC. X. J. was supported by a $\mathrm{PhD}$ studentship co-sponsored by the Chinese Scholarship Council and University College London. Z. F. and M. E. A. were supported by EPSRC Grant No. EP/M001946/1. A. C was supported by the European Research Council (ERC) under the European Union's Horizon 2020 research and innovation programme (grant agreement no. 682539/SOFTCHARGE). Via our membership of the UK's HEC Materials Chemistry Consortium, which is funded by EPSRC (EP/L000202), this work used the ARCHER UK National Supercomputing Service (http://www.archer.ac.uk). The authors acknowledge the use of the UCL Legion High Performance Facility (Legion@UCL). No competing financial interests have been declared.

\section{REFERENCES}

1. Nealson, K. H. Nature 2010, 463, 1033.

2. Summers, Z. M.; Fogarty, H. E.; Leang, C.; Franks, A. E.; Malvankar, N. S.; Lovley, D. R. Science 2010, 330, 1413.

3. Clarke, T. A.; Edwards, M.; Gates, A.; Hall, A.; White, G.; Bradley, J.; Reardon, C.; Shi, L.; Beliaev, A.; Marshall, M. J.; Wang, Z.; Watmough, N. J.; Fredrickson, J. K.; Zachara, J. M.; Butt, J. N. and Richardson, D. J. Proc. Natl. Acad. Sci. USA 2011, 108, 9384.

4. White, G. F.; Shi, Z.; Shi, L.; Z. Wang; Dohnalkova, A. C.; Marshall, M. J.; Fredrickson, J. K.; Zachara, J. M.; Butt, J. N.; Richardson, D. J.; Clarke, T. A. Proc. Nat. Acad. Sci. USA 2013, 110, 6346.

5. Edwards, M. J.; White, G. F.; Norman, M.; Tome-
Fernandez, A.; Ainsworth, E.; Shi, L.; Fredrickson, J. K.; Zachara, J. M.; Butt, J. N.; Richardson, D. J.; Clarke, T. A. Sci. Rep. 2015, 5, 11677.

6. Pirbadian, S.; Barchinger, S. E.; Leung, K. M.; Jangir, H. S. B.; Bouhenni, R. A.; Reed, S. B.; Romine, M. F.; Saffarini, D. A.; Shi, L.; Gorby, Y. A.; Golbeck, J. H.; ElNaggar, M. Y. Proc. Natl. Acad. Sci. 2014, 111, 12883.

7. Jensen, H. M.; Albers, A. E.; Malley, K. R.; Londer, Y. Y.; Cohen, B. E.; Helms, B. A.; Weigele, P.; Groves, J. T.; Ajo-Franklin, C. M. Proc. Natl. Acad. Sci. USA 2010, 107, 19213.

8. Leung, K. M.; Wanger, G.; El-Naggar, M. Y.; Gorby, Y. A.; Southam, G.; Lau, W. M.; Yang, J. ACS Nano 2013, 13, 2407.

9. Breuer, M.; Zarzycki, P.; Blumberger, J.; Rosso, K. M. J. Am. Chem. Soc. 2012, 134, 9868.

10. Breuer, M.; Rosso, K. M.; Blumberger, J. Proc. Nat. Acad. Sci. USA 2014, 111, 611.

11. Blumberger, J. Chem. Rev. 2015, 115, 11191.

12. Polizzi, N. F.; Skourtis, S. S.; Beratan, D. N. Faraday Discuss. 2012, 155, 43.

13. Pirbadian, S.; El-Naggar, M. Y. Phys. Chem. Chem. Phys. 2012, 14, 13802.

14. Strycharz-Glaven, S. M.; Snider, R. M.; Guiseppi-Elie, A.; Tender, L. M. Energy Environ. Sci. 2011, 4, 4366.

15. Zhang, Y.; Liu, C.; Balaeff, A.; Skourtis, S. S.; Beratan, D. N. Proc. Nat. Acad. Sci. USA 2014, 111, 10049.

16. Byun, H. S.; Pirbadian, S.; Nakano, A.; Shi, L.; ElNaggar, M. Y. ChemElectroChem 2014, 1, 1932.

17. Wigginton, N. S.; Rosso, K. M.; Lower, B. H.; Shi, L.; Hochella, M. F. Jr. Geochim. Cosmochim. Acta 2007, 71, 543.

18. Leys, D.; Meyer, T. E.; Tsapin, A. S.; Nealson, K. H.; Cusanovich, M. A.; Van Beeumen, J. J. J. Biol. Chem.

2002, 277, 35703 .

19. Harada, E.; Kumagai, J.; Ozawa, K.; Imabayashi, S.; Tsapin, A. S.; Nealson, K. H.; Meyer, T. E.; Cusanovich, M. A.; Akutsu, H. FEBS Letters 2002, 532, 333.

20. Fonseca, B. M.; Saraiva, I. H.; Paquete, C. M.; Soares, C. M.; Pacheco, I.; Salgueiro, C. A.; Louro, R. O. J. Biol. Inorg. Chem. 2009, 14, 375.

21. Futera, Z.; Blumberger, J. J. Phys. Chem. C 2017, 121, 19677.

22. Perdew, J. P.; Burke, K.; Ernzerhof, M. Phys. Rev. Lett. 1996, 77, 3865.

23. Oberhofer, H.; Blumberger, J. Angew. Chem. Int. Ed. 2010, 49, 3631.

24. Oberhofer, H.; Blumberger, J. Phys. Chem. Chem. Phys. 2012, 14, 13846.

25. Oberhofer, H.; Reuter, K.; Blumberger, J. Chem. Rev. 2017, 117, 10319.

26. Matyushov, D. V. J. Chem. Phys. 2013, 139, 025102.

27. Seyedi, S. S.; Waskasi, M. M.; Matyushov, D. V. J.

Phys. Chem. B 2017, 121, 4958.

28. Moser, C. C.; Keske, J. M.; Warncke, K.; Farid, R. S.; Dutton, P. L. Nature 1992, 355, 796.

29. Beratan, D. N.; Onuchic, J. N.; Hopfield, J. J. J. Chem. Phys. 1987, 86, 4488.

30. Beratan, D. N.; Balabin, I. A. Proc. Nat. Acad. Sci.

USA 2008, 105, 403. 


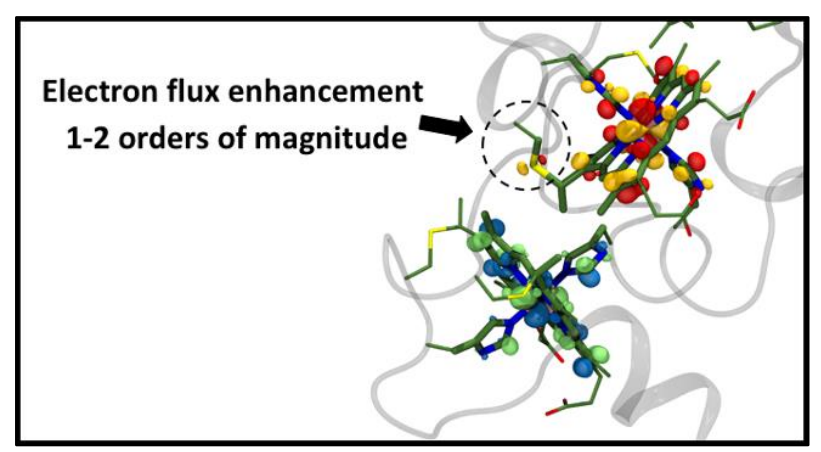

